\title{
VERA and East Asian VLBI network
}

\author{
Hideyuki Kobayashi ${ }^{1}$ \\ National Astronomical Observatory of Japan \\ 2-21-1、 Osawa, Mitaka、Tokyo, 181-8588, Japan \\ E-mail: hideyuki.kobayashi@nao.ac.jp
}

In East Asia, there are more than 20 VLBI stations, which are organized as their own country's network in Japan, Korea, and China. VERA was constructed in 2002 and aims precise astrometry by using 2-beam phase referencing technique in Japan. It is producing the results of proper motion and trigonometric parallax measurements for Galactic maser sources. Japan also has 13 station VLBI network including VERA. Korea has constructed 3 station VLBI network for millimetre VLBI with mutli-frequency phase referencing. China has four station VLBI network for satellite tracking and astronomy. From 2004, East Asian VLBI Network consortium prepares collaboration network of them. From 2011, science commissioning observations were started. In 2010, a new large correlator for EAVN was constructed. Science operation of EAVN is expected to start in 2013.

Resolving the Sky - Radio Interferometry: Past, Present and Future

Manchester, UK

April 17-20, 201

\footnotetext{
${ }^{1}$ H.Kobayashi
} 


\section{Introduction}

In the world, three major VLBI networks are working in the field of radio astronomy: VLBA, EVN, and LBA in USA, Europe, and Australia, respectively. In East Asia, new VLBI network is orgnaizing: East Asian VLBI Network(EAVN). In Japan, the first VLBI satellite: HALCA was lauched in 1997, and a space VLBI observation program was carried out. After it, new astrometry VLBI system: VERA was constructed in 2004, which is mainly aimed for proper motion and trigonometric parallax measurements for galactic maser sources. Some Japanese unviversities:Yamaguchi, Hokkaidou, Gifu and Ibaraki universities have VLBI stations and lead Japanese VLBI network with NAOJ and ISAS. In China, four VLBI stations: Shanghai, Urumqi, Miyun, and Kunnming was organized as CVN, which is mainly aimed for the lunar satellite tracking. C China has launched a series of lunar satellite since 2008. Also a new 65-m radio telescope has been constructed by Shanghai Astronomical Observatory. In Korea, new VLBI network with three 21-m radio telescopes was construted in 2008, which can make a multi-frequency observations for a kind of phase referencing VLBI. Then more than 20 VLBI stations are there in East Asia region. Moreover Taiwan has started a new 12-m submillimeter telescope project in Greenland.

\section{VERA project}

VERA has 20-m redio telesscope in Japan archipelago for phase referencing VLBI

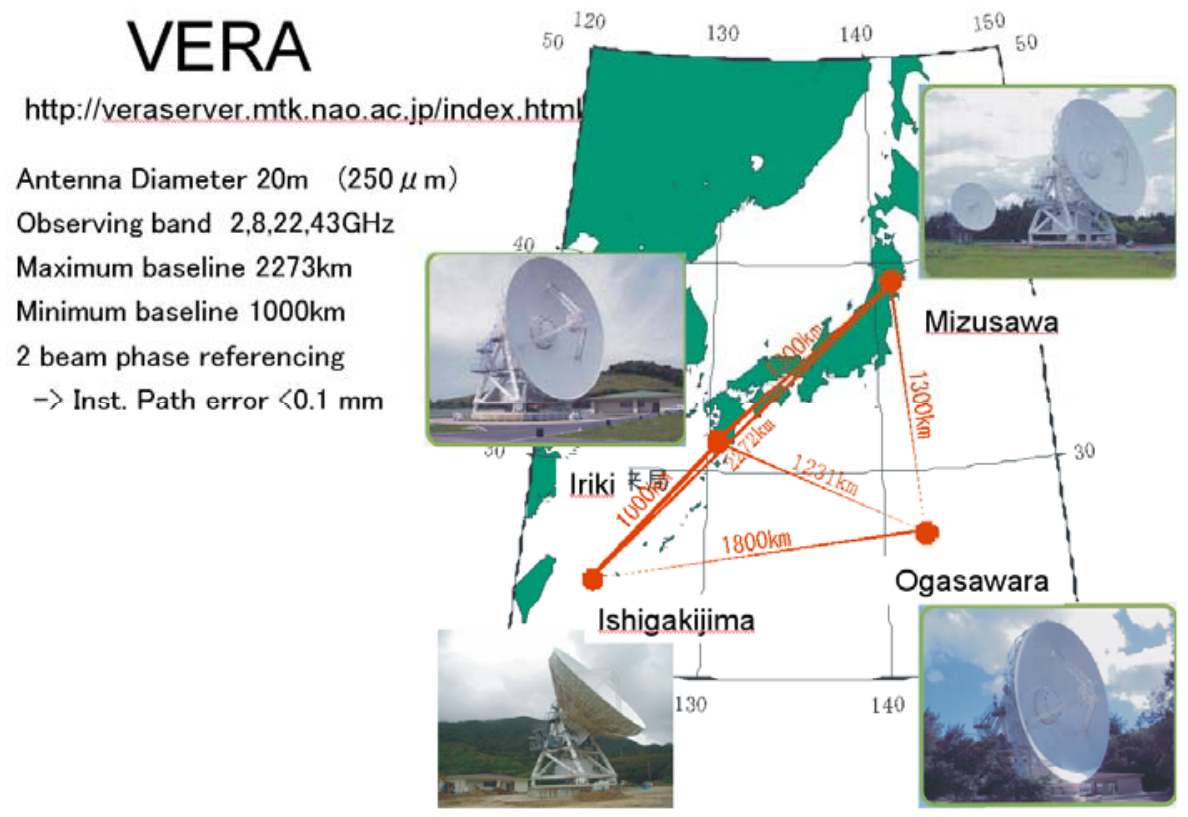

Figure 1. Station distribution and baseline length of VERA

Four VERA station are in Japan with around 2300 kilometer longest and 1000 kilometer shortest baselines. 
observations.(Kobayashi et,al, 2006) The array distribution is shown in Figure 1.

They have four receiver bands:2,8,22, and $43 \mathrm{GHz} . \quad 2$ and $8 \mathrm{GHz}$ receivers are used for geodesy VLBI observations. And 22 and $43 \mathrm{GHz}$ receivers are mainly used for maser source VLBI observations, which are bright and compact sources around star-forming molecular gas or envelop gas around evolved stars.

VERA has a two-beam observation system, which makes simultaneous pointing for two sources. Two receiver systems are equipped on the stewart platform, which are supported by 6 stiff jacks for each ones. It can move on the focal plane with 1.1 degree field of view. Then it can observe different objects with 2.2 degree separation at the maximum. A conceptional view of VERA two-beam system is shown in Figure 2. It makes perfect phase-

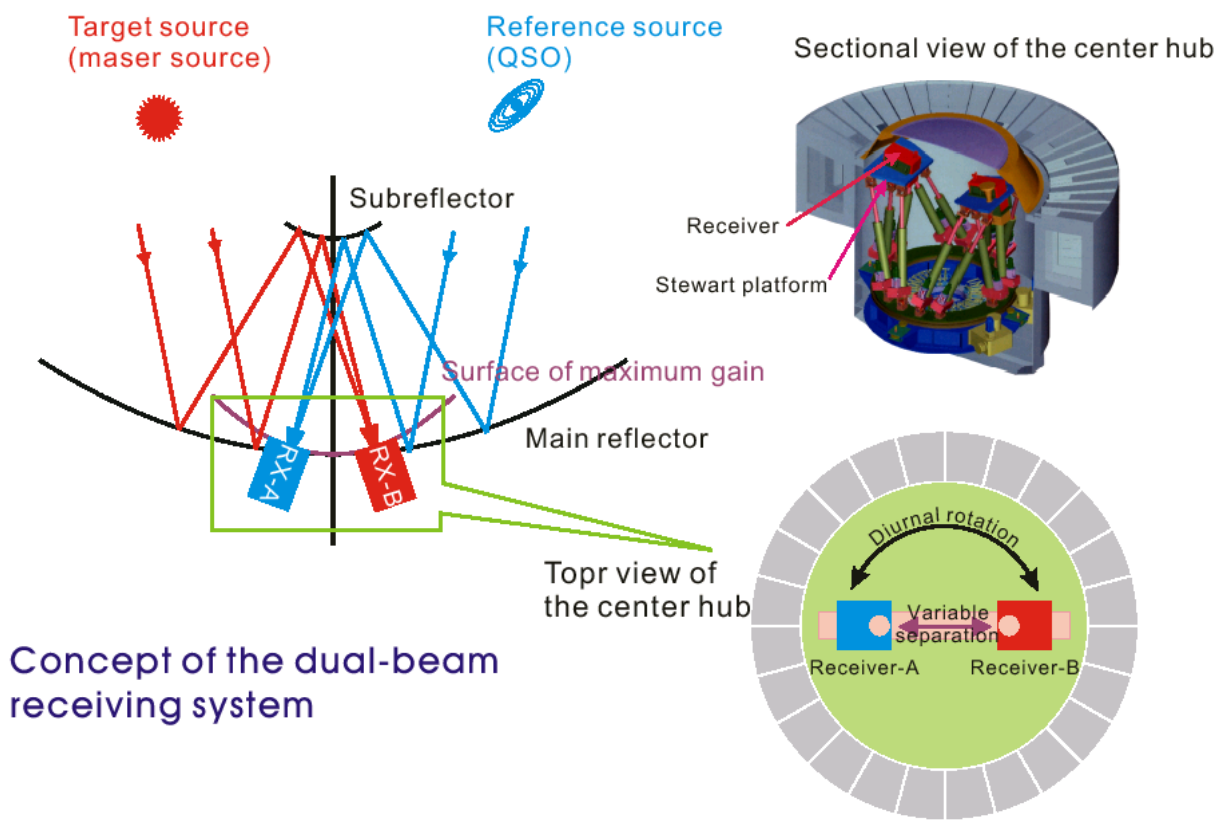

Figure 2 Concept of 2-beam system. Receivers are supported on the movable plates. They can move on the focal plane with 1.1 degree offset from the center of the field at the maximum.

referencing observations. A science goal of VERA is astrometry for the Galactic maser sources. In order it, $\mathrm{H} 2 \mathrm{O}$ and $\mathrm{SiO}$ maser sources in the Galaxy and backgrround AGN soureces are observed simultaneouly and relative phase of visibilities are used for positioning of maser soureces. Trigonomteric parallax and proper motion of each sources has been determined with less than $10 \%$ accuracy at most objects. From 2004, astrometry project has started Until the end of 2011, more than 30 sources has been determined the trigonometric parallaxes and proper motions, which is shown in Figure 3. And the distance between the Sun and the Galactic center is estimated as $8.1 \pm 0.4 \mathrm{kpc}$, which is smaller than the IAU recommendation.(Honma et.al, 2012) VERA has observed around a hundred objects to measure the trigonometric parallaxes and proper motions in a year. 
A huge earthquake:M9 attacked Japan on March 11, 2011. The origin of the earthquake is around 150 kilometer far from Mizusawa station. 20-m telescope in Mizusawa met sever damege of deformation of elevation driving gear on vertical plate. It was recovered by readjustment of the gear. Moreover Mizusawa was slipped around 3 meter by the earthquake.

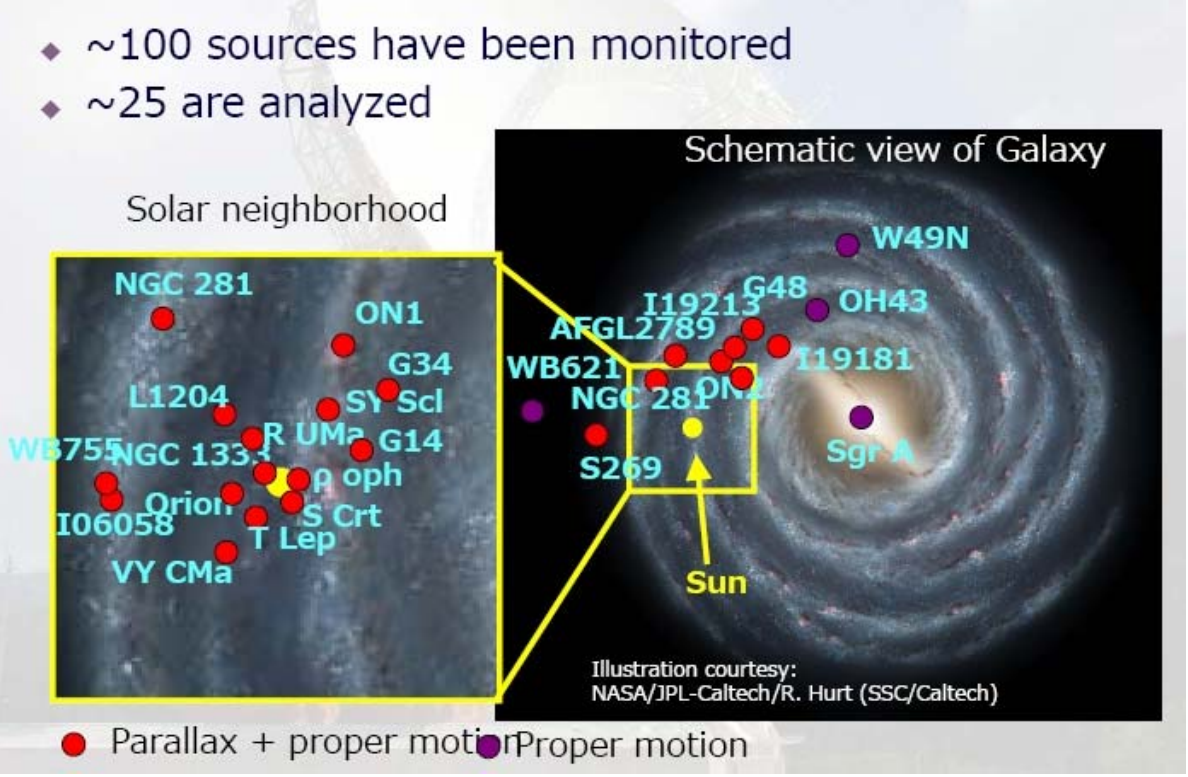

Figure 3: Object distribution of astrometry observation by VERA

Red dots show parallax and proper motion measured objects and purple dots

In order to make precise astronometry, station positions of each stations should be decided with several millimeter accurcy. VERA uses VLBI geodesy and GPS measurements for it. But after the earthquake, station positions of VERA stations were reset. We have started station position measurements again.

\section{Japanese VLBI network: JVN}

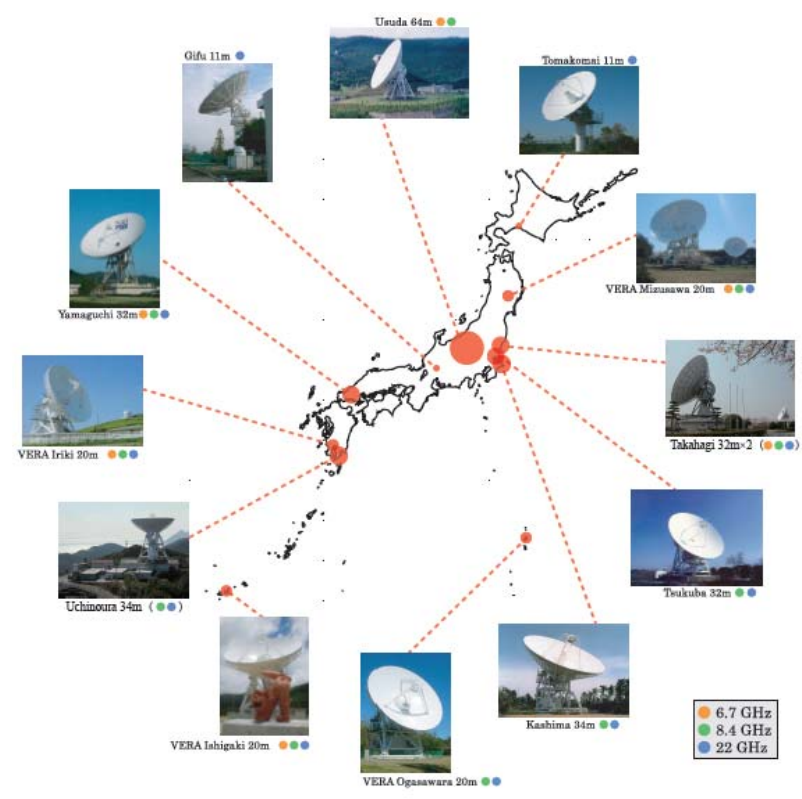

In Japan, 13 VLBI station are working for radio astronomy use including VERA. A 32-m telescope is operated by Yamaguchi University and two 32-m telescopes are operated by Ibaraki University. A 64-m telescope and 34-m telescope are operated by ISAS at Usuda and Kagoshima, respectively. Hokkaido University and Gifu University have 11-m telescope, respectively. NICT has 34-m

Figure 4; Station distribution of JVN. They have 6.7, 8, and $22 \mathrm{GHz}$ observation bands. 
telescope in Kashima and GSI has 32-m telescope in Tsukuba. Station distribution is shown in Figure 4. Maximum baseline length is around 2500 kilometers.

JVN has three observation bads: $6.7,8$, and $22 \mathrm{GHz}$. $6.7 \mathrm{GHz}$ observations aim for methanol maser objects. $8 \mathrm{GHz}$ observation aim for mapping of AGN and other continuum sources. $22 \mathrm{GHz}$ observation aim for water maser objetcs and AGN objects. Recently distributions of $6.7 \mathrm{GHz}$ class I mathenol maser sources are observed (Fujisawa et.al, 2012) They show 6.7GHz mathanol masers are associated with jet and disk structures. For the continuum observations, JVN can achive high dynamic range mapping with around 1000 .

\section{Korean VLBI network: KVN}

Korean Astronomy and Space science Institute:KASI has constructed a VLBI network with three 21-m telescopes.(Kim et al. 2004) Maximum baseline length is 480 kilometer and minimum is 135 kilometer. For millimeter wavelength, short baselines are important for AGN observations, because KVN $129 \mathrm{GHz}$ has comparable spatial resolution of VLBA $5 \mathrm{GHz}$. Figure5 shows the station distribution of KVN.

It has 22,43,86, and $129 \mathrm{GHz}$ receivers to observe $\mathrm{H} 2 \mathrm{O}$ and $\mathrm{SiO}$ maser sources and continuum soureces. KVN can observe with any bands of 22, 43, 86 and $129 \mathrm{GHz}$, simultaneouly, which makes multi-frequency phase referencing. Figure 6 shows the concept of multi-frequency receiver system, which has frequency-selective surfaces - low pass filters (LPFs). KVN can make SiO J=1-0, 2-1, 3-2 lines

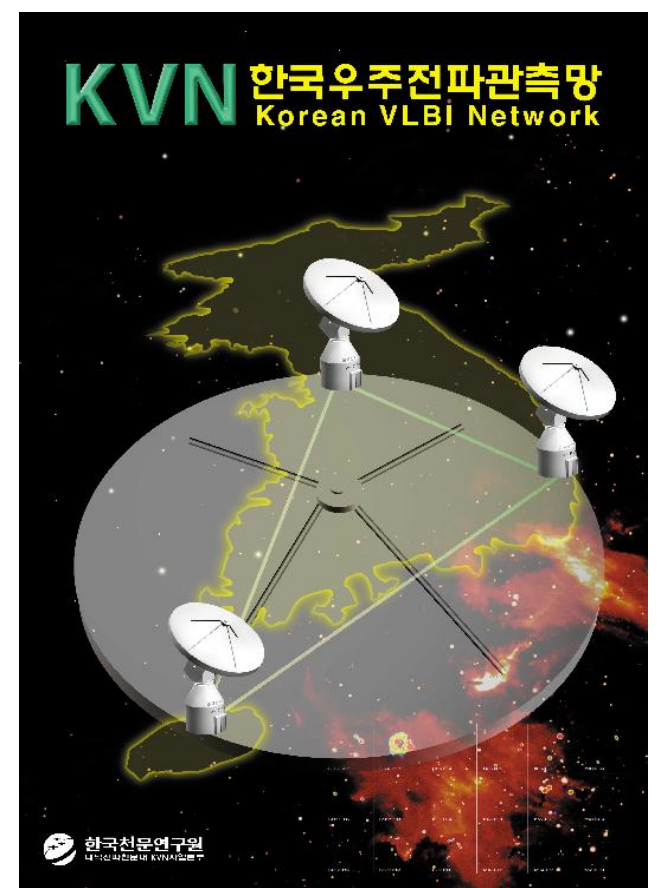

Figure 5: Station distribution of KVN Three stations are in Korean peninsula with 21-m diameter.(Kim, et al., 2004) observations around evolved stars, simultaneously. Monitering of different exciation SiO maser lines is important goal of KVN. Construction of three stations have been finished at the end of 2008. A new correlator is under developments. KVN is in science commissioning. 


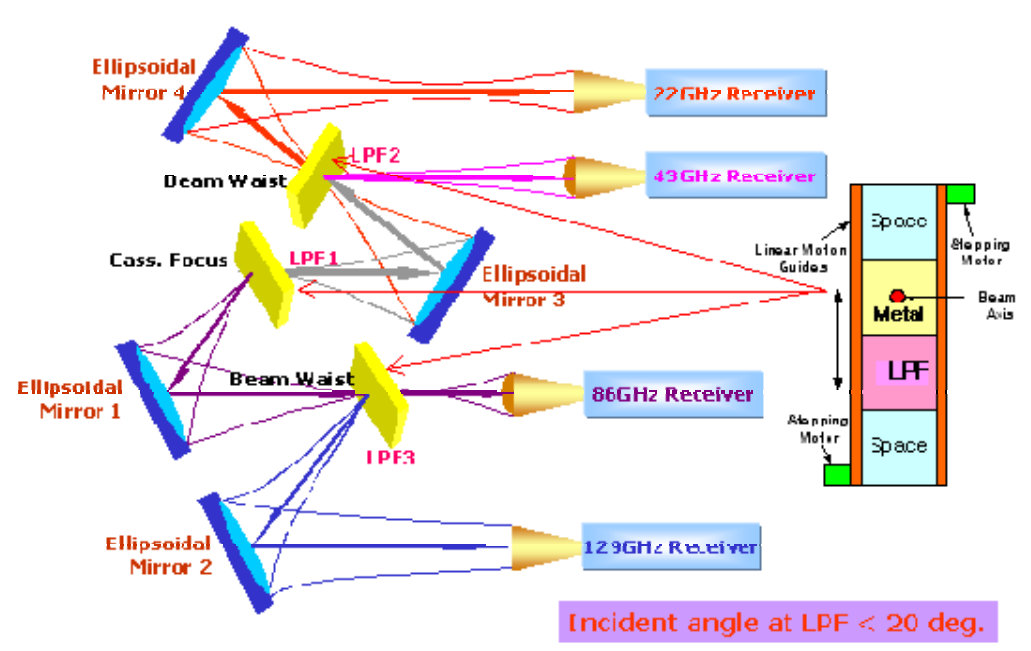

Figure 6: Conceptual view of KVN multi-frequency receivers.

LPFs transmit lower frequency signal and reflect higher frequency signal.(Kim et al., 2004)

\section{Chinese VLBI network: CVN}

China has four VLBI stations: Shaghai, Urumqi, Kunming and Miyun. Shanghai has a 25-m telescope with L. C. S/X, and K band receivers. And new 65-m telescope is under construction, which has active surface mechanism for panel adjustments and is expected to operate until Q band. Urmuqi has another 25-m telesope with L, C, $\mathrm{S} / \mathrm{X}$ and $\mathrm{K}$ band receivers. Shanghai and Urumqi stations are joining EVN. Kumimg 40-m telescope and Miyun 50-m telescope were constructed for lunar mission tracking in 2008, which have S/X receivers. Kunming Also developing new $\mathrm{L}$ and $\mathrm{C}$ band receivers. All four stations have Mk5b recording system. New correlator was constucted by Shanghai Astronomical Observatory, which can make semi-realtime correlation for satellite trackings. Chinese lunar satellites are navigated by CVN VLBI tracking. National Astronomical Observatory 
of China is constructing new 500-m telescope: FAST, which is ground-fixed type telescope like Arecibo 300-m telescope. It will be operated at L, C, and X bands.

\section{East Asian VLBI network: EAVN}

East Asian VLBI consortium was started at 2004 to organize the VLBI network in

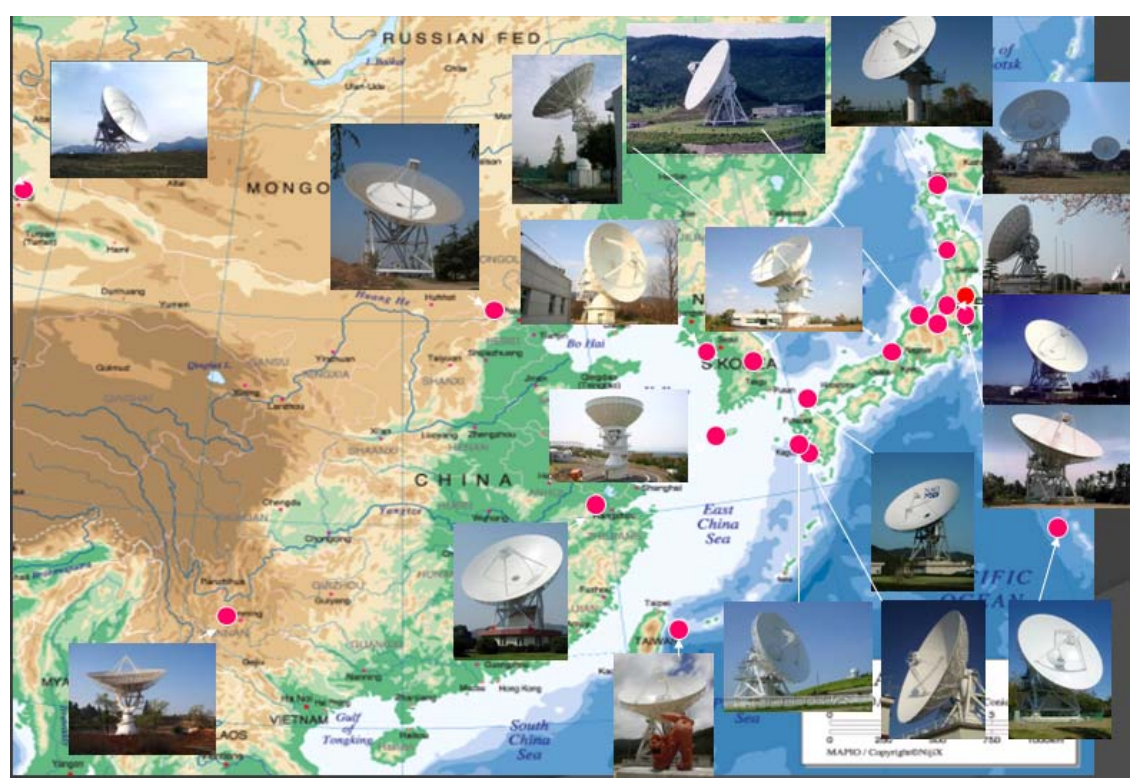

Fugure 8 Red dots show the position of EAVN stations, which is 20 with around $5000 \mathrm{~km}$ and $2500 \mathrm{~km}$ extent in East-West and North-South directions, respectively. east asia, which is under East Asia Core Observatory Association:EA COA. EACOA is an astronomy organization to activate collaboration among National Astronomica Observatory of Japan, Korean Astronomy and Space Science Institute, Academica Sinica Institute for Astronomy and Astrophysics, and National Astronomical Observatoruy of China. EAVN is a combined network of JVN, KVN, and CVN, which has more than 20 VLBI stations and is mainly operated at 8 and 22 GHz. The distribution of VLBI stations is shown in Figure $8 . \quad$ It is extended around 5000 $\mathrm{km}$ in east-west and $2500 \mathrm{~km}$ in northsouth directions.

In order to realize the EAVN, new large correlator facility is needed. NAOJ and KASI has collaborated to develop a new correlator of VLBI at

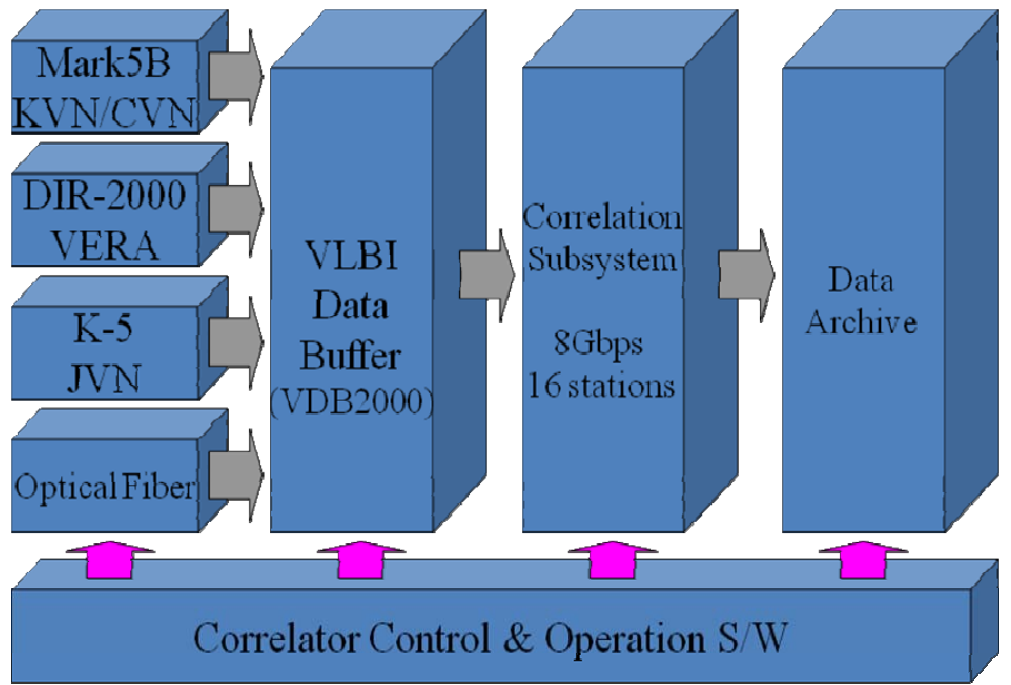

Figure 9 Block diagram of EAVN correlator It has a capability to input 16 stations with $8 \mathrm{Gps}$ date date per station. 
Daejon, Korea, which has a capability of 16 stations with 8Gbps per station data rate. It is possible to accept VLBI date from many kind of recorders with VSI format. In order to do it, disk data buffers are used. All VLBI data are transfered to them at first and correlated. Figure 9 shows the block diagram of the correlator system. Construction of the correlator was finished in 2010 and is in comissioing. It will start usual operation in 2012. NAOJ and KASI has an agreemnt for joint operations.

By the combined array of VERA and KVN, some test observations was carried out and good images show feasibility of it. Also methanol maser observations was carried out at $6.7 \mathrm{GHz}$ by JVN and Shanghai station. Such a comissioning observations will be carried out in 2012. And EAVN is expected to start science operations in 2013.

\section{References}

[1] H.Kobayashi, et al., 2008, A Giant Step: from Milli- to Micro-arcsecond Astrometry, Proceedings of the International Astronomical Union, IAU Symposium, Volume 248, p. 148-155

[2] K.Fujisawa, et al., 2012, Cosmic Masers - from OH to H0, Proceedings of the International Astronomical Union, IAU Symposium, Volume 287, p. 288-289

[3] M.Honma, et al., 2012, PASJ in press

[4] H.G.Kim, et al., . 2004, Proceedings of the 7th Symposium of the European VLBI Network on New Developments in VLBI Science and Technology.p281-283 